\title{
Confronting Arboviruses: María Guadalupe Guzmán MD PhD DrSc Director, Reference Center for Research \& Diagnosis Pedro Kourí Tropical Medicine Institute, Havana
}

\author{
Conner Gorry MA
}

The 1980s were a watershed for Cuban research in medicine and health: significant financing and material resources buttressed a strategy to improve population health through enhanced biopharmaceutical innovation and clinical best practices applied to Cuba's universal public health system. Redirecting research priorities and providing substantial public funding to tackle the top population health problems was a radical idea at the time, especially for a developing country like Cuba. Doing so has become a hallmark of Cuba's scientific achievements and approach ever since. Among the institutions exemplifying this strategy is the Pedro Kourí Tropical Medicine Institute (IPK). Founded in 1937 with a research mission dedicated to parasitology and transmission of known tropical diseases, it wasn't until the late Dr Gustavo Kourí Flores

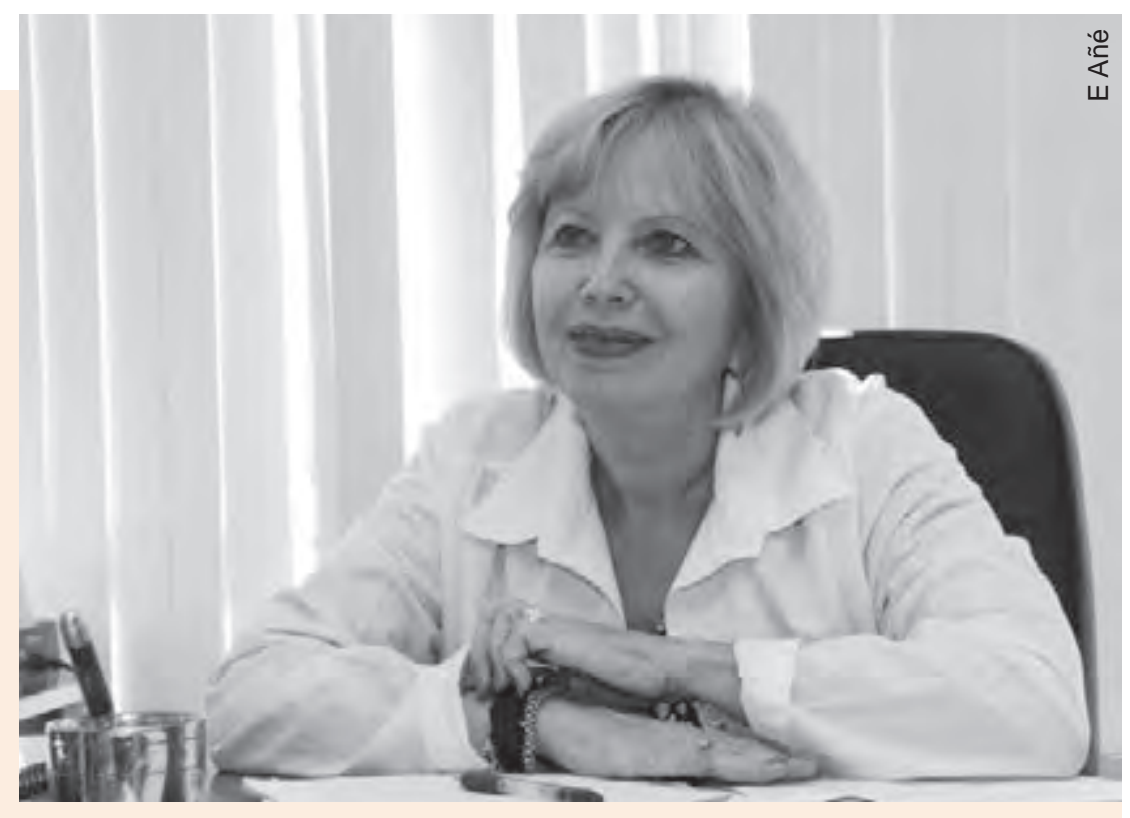
was appointed director in 1979 that IPK's core objectives and facilities were expanded to include a comprehensive teaching component, a state-of-the-art clinical hospital to treat tropical and other communicable diseases, and an international collaboration strategy to facilitate knowledge and technology transfer.

Today, IPK is Cuba's national reference center for diagnosis, treatment, control and prevention of communicable diseases and is a regional leader in applied research into socalled neglected diseases-usually diseases of the poor. With departments of parasitology, bacteriology, virology, pharmacology and more, it's a magnet for some of the country's most accomplished scientists-most of them women-and a major contributor to Cuba's portfolio of scientific products, research and publishing.

\section{MEDICC Review: The Institute was founded as a center for tropical disease research, but today has a much broader mandate. Can you explain what you and your colleagues do here?}

María Guadalupe Guzmán: When Dr Gustavo Kourí became director, he called IPK a sui generis institute-it's an institute that does research, but we do much more than just research. We have a clinical hospital and diagnostic laboratories; we conduct courses and seminars and so on. It's a necessarily complex structure because the work we do is complex.

Breaking it down, IPK is Cuba's national reference center for communicable diseases, divided into three centers: the hospital,
This interview with virologist Dr María Guadalupe Guzmán, director of IPK's Reference Center for Research and Diagnosis, is the third in MEDICC Review's series on outstanding Cuban women in science and medicine. Recognized as a leading expert in dengue research, Dr Guzmán is also director of the WHO/PAHO Collaborating Center for Dengue and its Control and was president of the Arbovirus Diagnosis Laboratory Network of the Americas (RELDA) from 2010 through 2018. Currently, she is president of the Cuban Society of Microbiology and Parasitology, directs IPK's Scientific Council that sets the Institute's research priorities, and is a distinguished professor and author. In 2016, she published Dengue (Editorial Ciencias Médicas, Havana), the most comprehensive collection of original Cuban research available on the topic. which receives patients on an ambulatory and inpatient basis here at our Havana headquarters; the Surveillance Center, located in Jagüey Grande (Matanzas Province) for monitoring and treating quarantined patients during a disease outbreak; and the Center for Research and Diagnosis, where I work, alongside 230 other scientists and professionals. We have molecular biologists, parasitologists, virologists, epidemiologists, bacteriologists, geographers, mathematicians, immunologists, entomologists and medical doctors dedicated to scientific research, diagnosis and surveillance of all types of communicable diseases.

The Center also has a department of social research, which is extremely important, especially for vector-borne diseases like 
dengue and Zika. Prevention and control of these diseases need community participation and collaboration, but how do you motivate people to practically support prevention efforts? How do we effectively inform and educate the public about possible threats and raise their awareness about control actions? Our social research department looks at all of these questions and analyzes how communities function, to encourage them to adopt lifestyles that better serve their health and the health of people around them. In my mind, this is a much more difficult area than traditional scientific research because you're dealing with individuals, families and communities.

MEDICC Review: You mention dengue and Zika-two arboviruses along with chikungunya - that have ravaged the region in recent years. What are priority research areas for your Center on these diseases?

María Guadalupe Guzmán: Dengue has a long history in Cuba. We experienced outbreaks of Dengue 1 in 1977-1978, Dengue 2 in 1981 and 1997 and of different strains afterwards, which is what most people remember. But dengue was in Cuba long before that. During the First War of Independence (1868-1878), Cuban troops fighting under Ignacio Agramonte against Spain referred to an illness they called "the white person's affliction," because white soldiers on both sides were much more likely to contract it than black soldiers. This medical-anthropological piece of data led one of our scientists to begin researching the genetic component of dengue epidemiology and resistance. This is an advanced, innovative project which holds promise for future prevention strategies.

Our interdisciplinary team of researchers is working on how people's movements influence the spread of dengue. Of course, international travel is a well-known factor in dengue transmission, but this research drills down to the neighborhood level. Based on the idea that people move, but the mosquito doesn't, if you have a person with dengue who lives in Marianao but works in Playa (two adjacent municipalities in Havana-Eds.), could we use this to predict where the disease might show up next? This research uses statistical projection models and identifies "hot spots"-areas where there is a concentration of cases and social determinants that might affect transmission. For example, standing water or uncollected garbage, improper household water storage, or high level of mobility amongst residents who live in a hotspot and work elsewhere might contribute to arbovirus infection rates.

A team of young entomologists is working on some intriguing larva research as well. In Cuba, we have a strong national surveillance network, as do several other countries, for patients who have already contracted dengue. But if we can identify disease-carrying mosquitoes at the larval stage, this will improve our projections and prevention.

For all our work it's important to use diagnostic algorithms to help forecast what we might face down the road. Obviously, you can't predict and control for every possibility, but the better prepared your diagnostic, prevention and control systems are, when unforeseen events do present themselves, you'll be better equipped to confront them. This is a strategy our entire institution follows, in coordination with the Ministry of Public Health and their priorities, and helped us when cases of Zika emerged, for example.
We're also working on a recombinant dengue vaccine in collaboration with the Genetic Engineering and Biotechnology Center (CIGB). This is in pre-clinical trials and is showing satisfactory results.

MEDICC Review: You're a well-known dengue expert. In practical terms, how did Cuba's long experience with dengue contribute to the nation's response to Zika?

María Guadalupe Guzmán: Cuba is a pioneer in standardized protocols and adheres to PAHO's Integrated Management Strategy for Arboviral Diseases, implemented in 2003. This requires systematizing everything related to prevention, control and response: vector control nationwide; laboratory diagnostics; surveillance technology; and case management. It also involves systematizing social aspects of prevention and diagnosis-our entire population participates in vector control, for example. Participation by our citizenry is one area in which we've improved thanks to the PAHO strategy.

It proved advantageous that these protocols were already in place when Zika and chikungunya appeared in Cuba. We were able to extend the protocols to these arboviruses that, like dengue, are transmitted by the Aedes aegypti mosquito. These are different diseases of course, but share some common denominators and from the point of view of surveillance and control, respond to the same strategy. This is also true for yellow fever, which we have to incorporate into our surveillance as well; we don't have yellow fever in Cuba but that doesn't mean that it can't occur here.

As director of the WHO/PAHO Collaborating Center for Dengue and its Control, I've worked intensively in Cuba's national network of diagnostic laboratories. To have effective prevention and control, you need standardized protocols and best practices in the labs responsible for the testing - this includes delivery and storage of reagents used in diagnosis, quality control of diagnostic kits and confirmation of results, as well as training of laboratory professionals. When Zika appeared, our experience with dengue allowed us to adapt the model to respond to this health threat. We established diagnostic laboratories according to WHO protocols and best practices for Zika in Santiago de Cuba (for the eastern region), Villa Clara (for the central region) and Havana (for the western region). As with dengue, all test samples are then transported to IPK for confirmation.

The objective of RELDA, the regional network of diagnostic laboratories, is to strengthen best practices and systematize and evaluate protocols in the Americas for prevention, control and surveillance of arboviruses. With communicable diseases like these, fragmented interventions and actions work against public health; everyone has to be on the same page and IPK's accumulated experience in the field has helped strengthen the quality and coordination of labs across the Americas region.

MEDICC Review: We're talking about diseases that thus far predominate in poor countries-contexts where diagnostic best practices and protocols might be financially prohibitive or where there's little political will to implement them.

María Guadalupe Guzmán: In some cases, a proper clinical exam can distinguish among dengue, chikungunya and Zika. But clinically, dengue has a very short cycle-from five to seven 
days. If it develops into severe dengue, that means you have a very small window to save that patient's life. This, and the cost of testing every suspected case for each arbovirus, makes a strong argument for clear, concise clinical guidelines, predictive algorithms, and consistent, coordinated epidemiological surveillance and analysis for distinguishing among the different diseases.

In 2009, WHO issued new global guidelines for diagnosis, treatment, prevention and control of dengue. Cuba played a fundamental advisory role in drafting these guidelines following participation in DENCO (International Dengue Control Study; a multi-country prospective clinical study), a joint project between IPK, the European Union and other institutions. It shed light on how clinicians might more effectively diagnose and treat dengue. One major recommendation we and our international colleagues made was to establish clinical guidelines related to warning signs-symptoms that tip off a doctor that

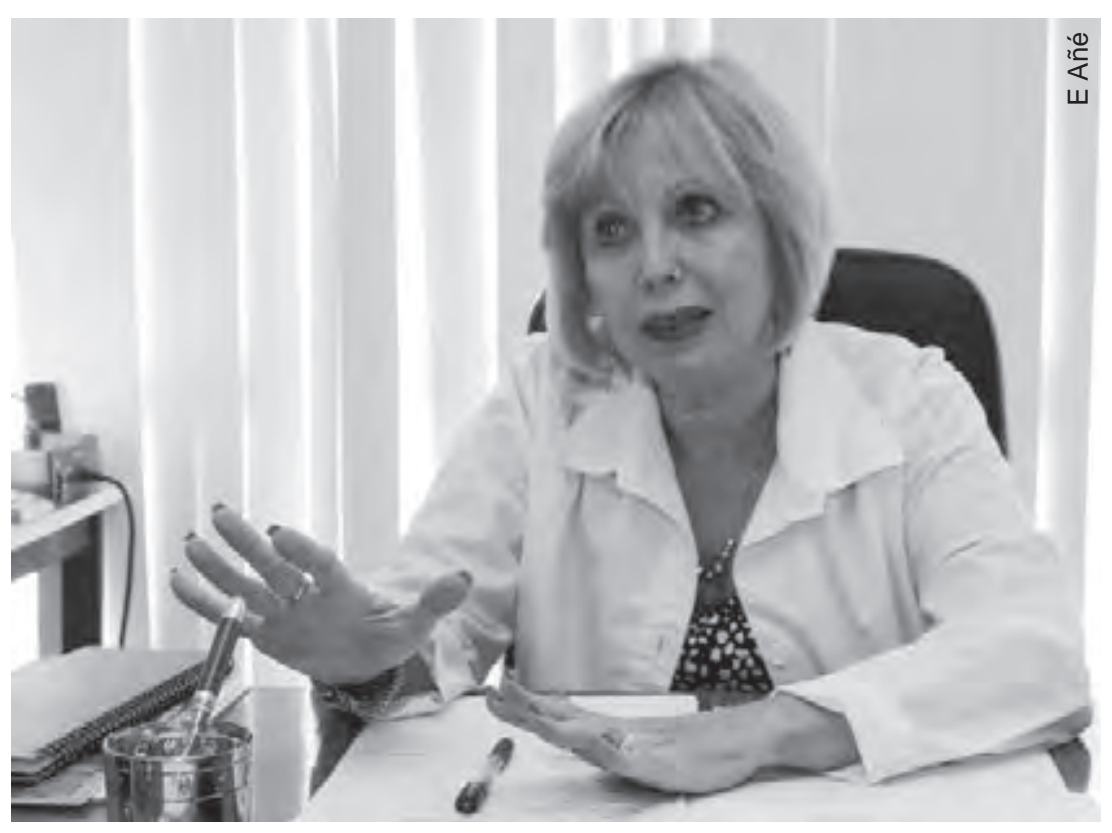
the patient may progress towards a more aggressive, and possibly fatal, form of dengue. Did your patient experience acute abdominal pain at the outset? Did they have persistent vomiting, experience fatigue or irritability, have bleeding from the mucous membranes? All of these serve as a kind of "dengue thermometer" for clinicians to identify those patients at risk of developing severe dengue.

There is no specific treatment for dengue. Rather, it's managed primarily by providing fluids, fluids, fluids!-juice, soup and of course water. The short cycle of dengue I mentioned, and the risk of some patients developing severe dengue, means early identification and intervention can save lives. I'm not a clinician, but in my opinion, the most valuable part of the new global guidelines are the recommendations related to clinical diagnosis. For years we've used clinical examination to look for warning signs, which in our experience can be life-saving.

Cuban experts worked closely with PAHO and other member countries when chikungunya and Zika emerged to draw up guidelines and informational materials to assist clinicians to distinguish among these three arboviruses-but of course, this is most effective when supported by a viable public health system, including a certified network of labs to confirm diagnoses.

\section{MEDICC Review: IPK is a WHO/PAHO Collaborating Center for Dengue and its Control and is active regionally and globally with other entities and organizations. Can you talk a little about these collaborations?}

María Guadalupe Guzmán: IPK has two WHO/PAHO collaborating centers: one for tuberculosis and the other for dengue. I head up the latter. We were first granted collaborating center status in 1996, when we were the reference center for virology. In 2005, we evolved into the reference center for dengue and were re-evaluated and certified. Every collaborating center has to adhere to protocols and best practices established at the global level by $\mathrm{WHO}$. Maintaining this status requires a comprehen- sive WHO/PAHO evaluation every four years, and we've been recertified each time. In our case, we are evaluated according to guidelines related to clinical practices, emergency and vector response, and laboratories-including diagnostic and surveillance components.

We have ongoing collaborations with European institutions and are currently part of the Zika Alliance-an EU research project investigating Zika in pregnant women-and Zika Plan, another EU joint project evaluating diagnostic kits for this arbovirus. I'm also co-coordinator with Swiss colleagues of a project designed to strengthen and expand our diagnostic labs for Zika. And then there's the International Course on Dengue.

\section{MEDICC Review: In arbovirus circles, this IPK International Course is widely known. What does it entail?}

María Guadalupe Guzmán: A lot of work! After each course, I'm ready for a year's sabbatical-but that's one of the challenges of my work: lots to do and too little time. The International Course on Dengue, Zika and Other Emerging Arboviruses is a biennial event that IPK first hosted in 1987 with half a dozen students. I was one of them. This year will be our 16th edition, and of course enrollment has grown significantly.

The two-week course is divided into two modules, theoretical and practical. The course goal is to bring together leading experts and share the latest scientific, research and clinical developments related to dengue and other arbovirusesincluding vaccine development, clinical practices, social factors, diagnosis and prevention. We always have robust participation from Latin America and Europe and we've hosted African colleagues here too, though the prohibitive cost of international travel is a limitation. Despite the current US administration, which makes everything related to Cuba more challenging, we have many colleagues from the USA coming this year, including experts from the National Institutes of Health, Johns Hopkins Schools of Medicine and Public Health and University of California Berkeley, among others. 
MEDICC Review: Looking at the roster of international participants, it appears only $25 \%$ are women. Yet, one of the striking things about Cuban science, medicine, research and technology is the presence of women-as innovators, investigators and leaders. Can you comment on the role of women at IPK?

María Guadalupe Guzmán: Most of the researchers, scientists and technicians at the Center for Research and Diagnosis are women-the hospital is more gender-balanced. But our Center attracts many graduates from the University of Havana's Biology Department where the majority of students are women.
Parasitology, virology, bacteriology, epidemiology-we have women doing all kinds of innovative research in these fields here. Women also predominate in vaccine development and they are leading our research on malaria, a disease not present in Cuba but a major global health threat, nonetheless. And finally, our professors, who form the core of our education here, are mostly women.

My granddaughter celebrated her first birthday on Cuba's National Science Day, January 15th-so, at the rate we're going, who knows? Maybe she'll become one of Cuba's greatest scientists! -1 - 